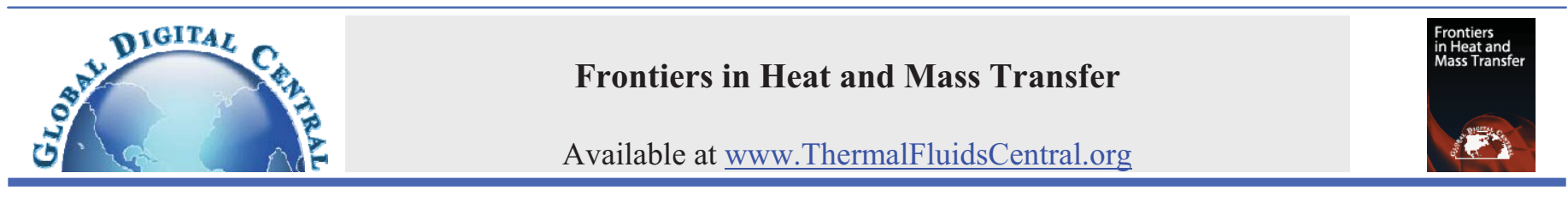

\title{
DOUBLE DIFFUSION EFFECTS ON CONVECTION IN FLOW ON VERTICAL PLATE IMBEDDED IN POROUS MEDIA
}

\author{
Z. Aouachria ${ }^{\mathrm{a},{ }^{*}}$ F. Rouichi ${ }^{\mathrm{a}}$, D. Haddad ${ }^{\mathrm{b},}$ \\ ${ }^{a}$ Laboratory of Applied Energetic Physics, University of Batna, Algeria \\ ${ }^{b}$ Institute of Institute of hygiene and security, University of Batna, Algeria
}

\begin{abstract}
Natural convection flow past a vertical porous plate in a porous medium is studied numerically, by taking into account the Dufour and Soret effects. The similarity equations of the problem considered are obtained by using usual similarity technique. This system of ordinary differential equations, which are solved numerically by using the Nachtsheim -Swigerst hooting iteration technique together with a sixth order Runge-Kutta integrations scheme. The results show that Soret and Dufour effects do not appreciably influence the velocity, temperature and concentration fields, but rather only tend to increase the mass and energy flux due to the added contributions.

Keywords: Free convection porous, media numerical simulation, similarity analysis.
\end{abstract}

\section{INTRODUCTION}

The free convection flows arising out of combined buoyancies due to the thermal and mass transfer in porous media are of $\mathrm{q}$ capital importance because of the fundamental nature of the problem and broad range of their applications relating to the manufacture and industrial process such as the geothermal systems, fibro, storage of the nuclear products, the dispersion of chemical contaminate. The combined heat and mass transfer in porous media has attracted considerable attention in the last time, because several applications of practical interest can be found in some industrial processes such as food processing and float glass production and its many important engineering and geophysical applications mentioned above Pilkington (1969).

The energy flux includes contributions due to a temperature gradient (Fourier heat conduction), concentration gradient (Fick diffusion) and a term which accounts for the energy transport as a result of each species having different enthalpies (species interdiffusion). The mass flux can be consisted of terms which are due to a concentration gradient (Fickian diffusion), temperature gradient (Soret diffusion), pressure gradient (pressure diffusion) and a term which is accounted by external forces affecting each species by a different magnitude.

Soret mass flux and the Dufour energy flux become significant when the thermal diffusion factor, the temperature and concentration gradients are large, Weaver and Viskantar (1991). These transfers, due to the formation of a concentration gradient induced by a temperature gradient are known as the Soret effect or thermodifusion. This effect was studied in gases in 1879 by C. Soret, is used for the separation of isotopic mixtures when it comes to weight molecules close such that of a molecular weight of the hydrogen and the helium or the average weight of (H2, air). The opposite effect, says Dufour effect, was found to be of considerable magnitude such that it cannot be overlooked, Mahmud and Nazmul (2007). The Dufour effect is the existence of a heat flow due to a concentration gradient, was studied in 1872 by L. Dufour.

A large number of papers have been published dealing with natural convection in a horizontal layer, induced by either horizontal or vertical temperature gradients, but very few have deal with the more general situation of inclined temperature gradients. Weber (1973) considered the problem of thermal convection with horizontal temperature gradients in a viscous fluid. Weber (1974) analyzed the effect of horizontal and vertical thermal gradients on convection in a porous medium. Bejan and Khair [1985] use the Darcy law to study the flow characteristics in the boundary layer, which is caused by thermal and concentration gradients. Lai et al have re-examined this convection along a vertical wall with constant heat and mass flow with the wall injection.

the Soret and Dufour coefficients give rise to interaction between the thermal and solute fields even when the fluid is at rest. However, it is well known that the Soret coefficient has a considerable effect on the convection process in liquids, whereas the literature survey reveals that the Dufour effect can be negligible in liquids, but it plays a prominent role in gaseous mixtures. Hurle and Jakeman (1971) have analyzed theoretically the Soret effect on the Rayleigh-Jewry problem neglecting the Dufour coefficient and have shown that stable solutions could occur owing to this effect in water-methanol mixtures when they are heated from below. They consider thermal diffusion induced by horizontal thermal gradient (in a strictly fluid cell). we also know that flows arising from differences in concentration have great significance not only for their own interest but also for the application to the geophysics, aeronautics and engineering.

In light of the above applications, many researchers studied effects of mass transfer in free convection on magnetohydrodynamics (MHD); as Raptis and Kafoussias (1982), Rahman and Sattar (1999), Yih (1999), Aboeldahab and Elbarbary (2001), Megahead et al. (2003) and Kim (2004). Very recently, Postelnicu (2004) studied numerically the influence of a magnetic field on heat and mass transfer by natural convection from vertical surfaces in porous media considering Soret and Dufour effects. The range of free convective flows that can occur in nature and in engineering practice is very large and has been extensively considered by Jaluria (1980) ans others. Recently, further work has appeared in the literature detailing nonlinear effects, the effect of different boundary conditions and flow with nit cavities, the reader is referred to Nield and Bejan (2006) for further discussion.

" Corresponding author Email :aouachria2001@gmail.com 


\section{MATHEMATIC MODELLING}

We consider a coupled heat and mass transfer by the mixed free forced convection. The flow of viscous incompressible fluid, over a semi infinite vertical porous plate embedded in porous media, is assumed steady and has a two-dimensional character. The flow is supposed to be in the $\mathrm{x}$ direction, which is taken along the vertical plate in the upward direction and the y axis is taken to be normal to the plate see Fig. 1. We consider also that the surface of plate is maintained at $T_{w}$ and a constant concentration $C_{w}$, which are higher than $\mathrm{T}_{\infty}$ and $\mathrm{C}_{\infty}$, far away the flat surface. The free stream velocity $U \infty$ is parallel to the vertical plate and is assumed constant. The coordinate system and the flow configuration are shown in Figure1.
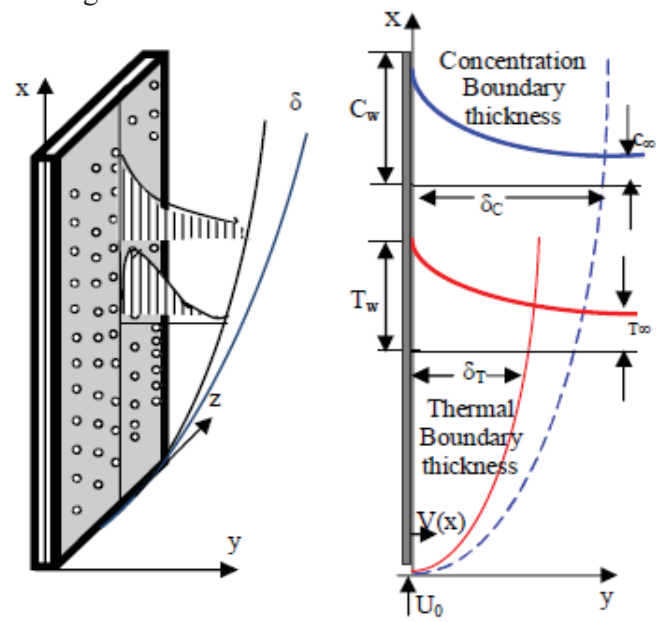

Fig. 1 Physical model and flow configuration

Using the Brinkman model jointly with the Boussinesq and boundary layer approximations, the governing equations relevant to the problem are as follows:

$$
\begin{aligned}
& \frac{\partial u}{\partial x}+\frac{\partial v}{\partial y}=0 \\
& u \frac{\partial u}{\partial x}+v \frac{\partial u}{\partial y}=v \frac{\partial^{2} u}{\partial y^{2}}+g \beta\left(T-T_{\infty}\right)+g \beta_{c}\left(C-C_{\infty}\right)-\frac{v}{k} u \\
& u \frac{\partial T}{\partial x}+v \frac{\partial T}{\partial y}=\alpha \frac{\partial^{2} T}{\partial y^{2}}+\frac{D_{m} K_{T}}{C_{s} C_{p}} \frac{\partial^{2} C}{\partial y^{2}} \\
& u \frac{\partial C}{\partial x}+v \frac{\partial C}{\partial y}=D_{m} \frac{\partial^{2} C}{\partial y^{2}}+\frac{D_{m} K_{T}}{T_{m}} \cdot \frac{\partial^{2} T}{\partial y^{2}}
\end{aligned}
$$

Here, $u$ and $v$ are the velocity components in the $x$ and $y$ directions respectively, $v$ is the cinematic viscosity, $g$ is the gravitational force due to acceleration, $\rho$ is the density, $\beta \mathrm{T}$ and $\beta \mathrm{m}$ are thermal and mass coefficient of volume expansion respectively. $T, T_{w}$ and $T_{\infty}$ are the temperature of the fluid inside while $\mathrm{C}, \mathrm{C}_{\mathrm{w}}$ and $\mathrm{C}_{\infty}$ are the corresponding concentrations. Also, $\pi$ is the permeability of a porous medium, $\alpha$ is the thermal diffusivity, $D m$ is the coefficient of mass diffusivity, $c p$ is the specific heat at constant pressure, $T m$ is the mean fluid temperature, $k_{T}$ is the thermal diffusion ratio and $c_{s}$ is the concentration susceptibility. The boundary conditions, for the model, are the thermal boundary layer, the plate temperature and the fluid temperature in the free stream, respectively and can be written as:

$\left\{\begin{array}{l}u=U_{0}, \quad v=+v_{0}(x), \quad T=T_{w}, C=C_{w}, y=0 \\ u=0, v=0, \quad T=T_{\infty}, C=C_{\infty}, y \rightarrow \infty\end{array}\right.$

\section{SIMILARITY ANALYSIS}

Making use of the following similarity transformation

$$
\begin{aligned}
& \eta=y\left(\frac{U_{0}}{v x}\right)^{\frac{1}{2}}, \psi=\sqrt{v x U_{0}} f(\eta) \\
& \theta(\eta)=\left(T-T_{\infty}\right) /\left(T_{w}-T_{\infty}\right) \\
& \phi(\eta)=\left(C-C_{\infty}\right) /\left(C_{w}-C_{\infty}\right)
\end{aligned}
$$

The above partial differential equations are transformed into ordinary differential equations

$$
\begin{aligned}
& \frac{1}{2} f f^{\prime \prime}+f^{\prime \prime \prime}+g_{s} \theta+g_{c} \phi-\frac{1}{D_{a} R_{e}} f^{\prime}=0 \\
& \theta^{\prime \prime}+\frac{1}{2} P_{r} \theta^{\prime} f+P_{r} D_{u} \phi^{\prime \prime}=0 \\
& \phi^{\prime \prime}+\frac{1}{2} S_{C} \phi^{\prime} f+S_{C} S_{r} \theta^{\prime \prime}=0
\end{aligned}
$$

along with the boundary conditions

$$
\left\{\begin{array}{l}
f^{\prime}=1, f=f_{w}, \theta=1, \phi=1 \quad \eta=0 \\
f^{\prime}=0, \quad \theta=0, \phi=0 \quad \eta \rightarrow \infty
\end{array}\right.
$$

\section{RESULTS AND DISCUSSION}

The equations (8)-(10) constitute a set of ordinary differential equations, the solutions of which should unfold the characteristics of the problem under consideration. These equations under the boundary conditions (11) are solved numerically by using the NachtsheimSwigert (1969) shooting iteration technique together with a sixth-order Runge Kutta integration scheme. Computations were first performed the value of Schmidt number $\mathrm{Sc}=0.22$ has been chosen to represent hydrogen at approximate $\mathrm{T}_{\mathrm{m}}=25^{\circ} \mathrm{C}$ and $1 \mathrm{~atm}$. The values of Grashof number $\mathrm{Gr}$ and modified Grashof number $\mathrm{Gm}$ are taken to be both arbitrary and positive and negative, since these values represent respectively, cooling and heating of the plate. Finally, the values of Soret number Sr and Dufour number Df are chosen in such a way that their product is constant, table 1 . The values of suction parameter are taken arbitrary and mass and thermal floatability parameters are constant, corresponding to $g_{s}=1$ and $g_{c}=0.2$. In comparing our results with those reported by (MS Alam, et al 2006), we see that the variation of temperature profile and concentration for different values of Df and Sr shows a good agreement between them.see Fig. 2.
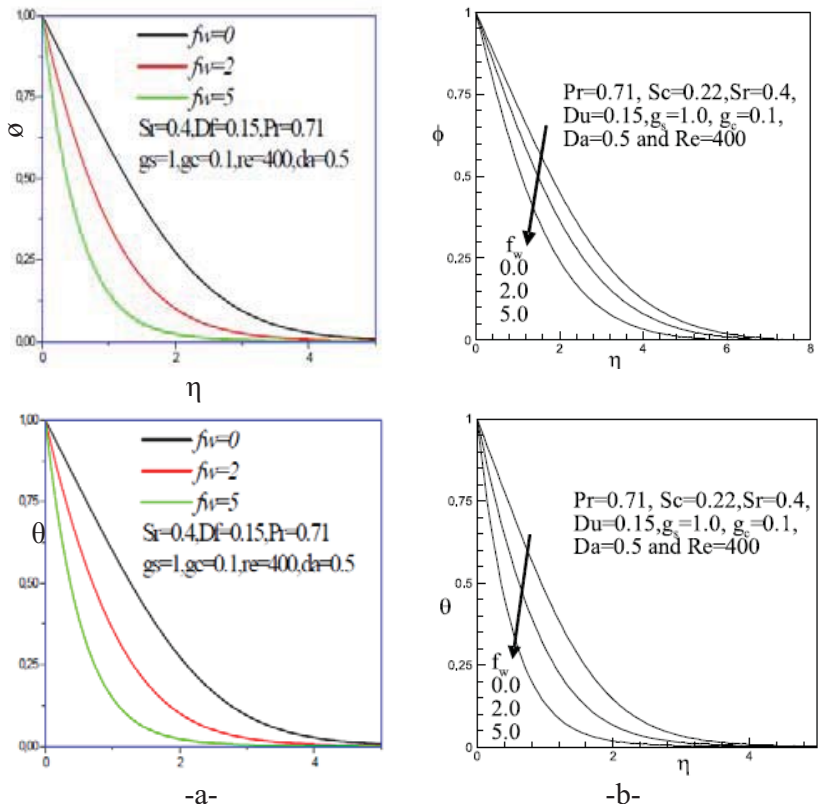

Fig. 2 comparison the $Q$ and $\theta$ of the present study $-a-$ and M.S. Alam, et al 2006, results -b-. 
Table 1 numerical values of Df and $\mathrm{Sr}$

\begin{tabular}{|l|l|l|l|l|l|l|}
\hline Sr & 2.0 & 1.6 & 1.2 & 0.8 & 0.4 & 0.1 \\
\hline Df & 0.03 & 0.037 & 0.05 & 0.075 & 0.15 & 0.6 \\
\hline
\end{tabular}

Table 2 air thermo physics parameters

\begin{tabular}{|l|l|l|l|l|l|l|}
\hline$\Gamma, \mathrm{K}$ & $\nu\left(\mathrm{m}^{2} . \mathrm{s}^{-1}\right)$ & $\rho\left(\mathrm{Kg} / \mathrm{m}^{3}\right)$ & $\mu(\mathrm{Pa} . \mathrm{s})$ & $\mathrm{Pr}$ & $\beta_{T}(1 / \mathrm{K})$ & $\beta_{C}\left(\mathrm{~m}^{3} \cdot \mathrm{k}\left(\mathrm{g}^{-1}\right)\right.$ \\
\hline 25 & $1.55 .10^{-5}$ & 1.184 & $0.0183 .10^{-3}$ & 0.707 & 0.00335 & -0.844 \\
\hline
\end{tabular}

\subsection{Dufour and Soret effects on velocity, concentration and temperature.}

Figure 2 shows the dimensionless velocity, temperature and concen-tration profile for different Dufour and Soret number values. In Fig. 2a, we observe that the velocity profile remains almost invariant for different values of Soret number. This explains that the Soret effect on the dynamic boundary layer is insignificant. However, we can note an influence regardless of this profile in two areas, the first locates the maximum of the profile corresponding to the interval $\eta=0.4-1.05$ and the second is localized in the low range equal to $\eta=2.1-4.7$. The Fig. $2 \mathrm{~b}$ shows that velocity increases when Dufour number increasing. Indeed, we note that the velocity increases near the wall where the temperature is higher but it decreases rapidly when one moves away from the wall. This is of course also to the suction caused by the inlet velocity $\mathrm{U}_{0}$. The Dufour number influence on velocity profile more pronounced as the Soret number. Note you get free flow, that is, outside the dynamics boundary layer for the value close to $\eta=6$.

We note, on the Fig. 2c, that the influence of the Soret effect, on the concentration profile, is more significant contribution than the Dufour effect that show in Fig. 2d. We observe that quantitatively, when $\eta=2$ and $\mathrm{Sr}$ decreases from 2.0 to 1.42 , there is $8.16 \%$ increase in the temperature value and $33.6 \%$ in the concentration value. While the number of Soret favorites the concentration increasing. But in Fig. $2 \mathrm{~d}$, there is the opposite phenomenon, i.e. the increase in concentration is achieved with fewer Dufour. So we can conclude that these two effects on the mass transfer are antagonistic. As we notice again that this increase in concentration with dimensionless number of Dufour is insignificant and in many cases it was admitted that the Dufour effect on the mass transfer is negligible. One can interpret the scope of the variable considered, where the concentration gradient becomes almost zero, as the thickness of the boundary layer of concentration. While it is clear that this thickness is larger than the boundary layer dynamics and thermal thicknesses. There, Fig. 2 expresses the correlation between the boundary layers of concentration, and thermal dynamics.

Figure $2 \mathrm{e}$ and $2 \mathrm{f}$ show the temperature field for different values of the Soret and Dufour numbers. It was found that the temperature profile remains almost invariant for different values of Soret number. In Fig. $2 \mathrm{f}$, we observe that the influence of the number of Dufour appears only area that stretches interval $\eta=[1.13-5]$ but beyond, the temperature decreases with the decrease in Dufour. We note that the Dufour number affects the thermal boundary layer thickness as demonstrated, Fig. 2f, for Velocity, concentration and temperature profiles versus $\eta$ for different values of pair (Df, Sr), Aouachria et al (2010).

\subsection{Influence of the parameter of suction, $f_{w}$, profiles of} velocity, concentration and temperature.

Figure 3 represents the velocity, concentration and temperature variations for different values of suction parameter, $f w$. The numerical results for velocity profiles depending on this parameter is shown in Fig. 3a, where we notice the significant decrease in the rate with increasing suction parameter indicating the usual phenomenon of stabilization of the growth of boundary layer which leads aspiration. Also in Fig. 3b and Fig. 3c, the same phenomenon is observed on temperature and concentration profiles. While sucking the liquid particles slowed near the porous wall by reducing the growth of the dynamics boundary layer as well as the thermal boundary layer and concentration.

\subsection{Dufour and Soret effects on the friction coefficient}

It has traces in Fig. 4, the friction coefficient based on the number of Dufour and Soret. We note in Fig. 4a that, whatever the value of many of Soret number, the friction coefficient increases linearly with increasing Dufour number. For Df $<0.15$, friction coefficient increases with increasing Soret number, but at this value, Sr does not affect the friction coefficient. But for $\mathrm{Df}>0.13$, the friction coefficient decreases with increasing Soret number. In Fig. $4 \mathrm{~b}$ for $\mathrm{Df}=0.03$, the coefficient of friction increases slightly with increasing number of Soret. By cons, for higher values of Df we see the opposite effect. In fig.10, the analysis highlights the importance of the Dufour effect is more prominent than the Soret effect. There is an invariance of the coefficient of friction for the value of the number of Dufour equal to 0.15 .
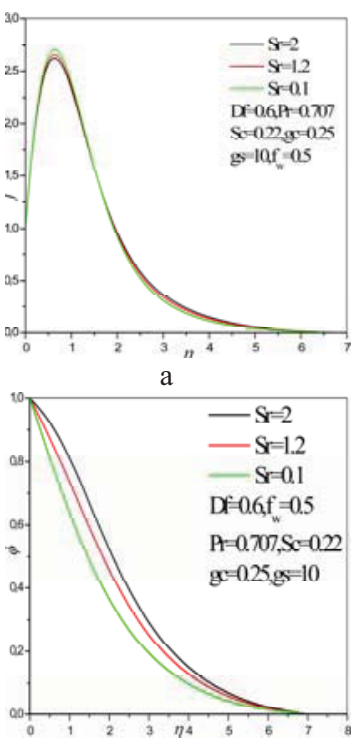

$\mathrm{c}$

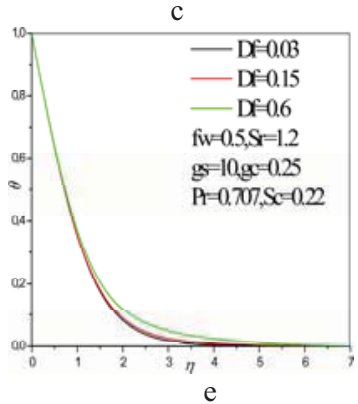

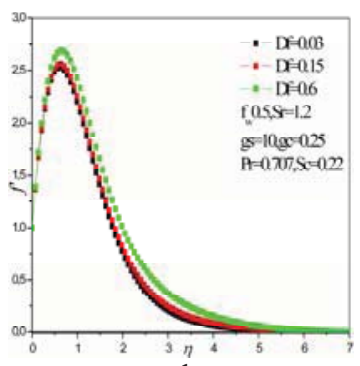

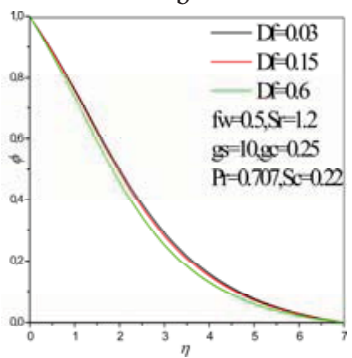

d

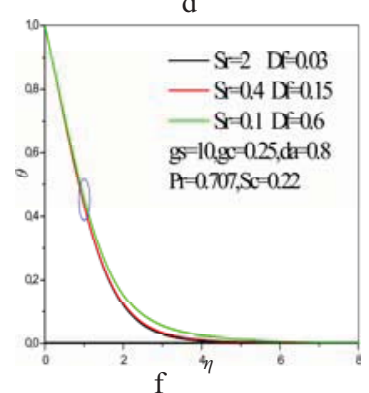

Fig. 2 Dufour and Soret effects on the velocity, $f^{\prime}$, temperature, $\theta$, and concentration, $\varphi$.

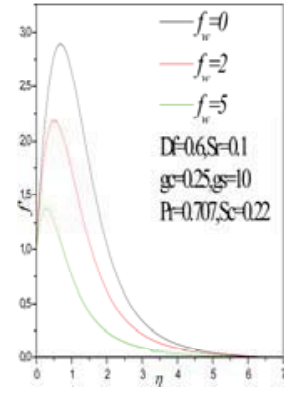

a

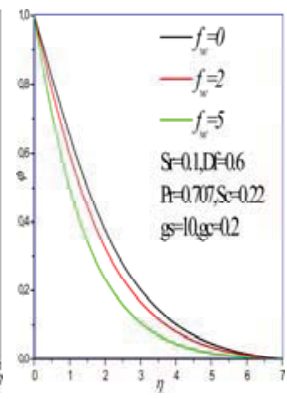

b

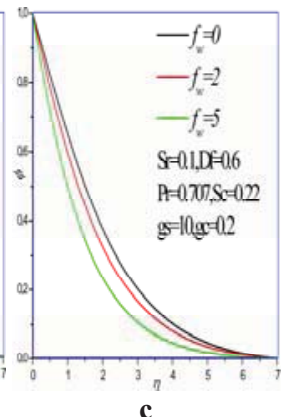

Fig. 3 Velocity, a, concentration, b and temperature, c, profiles as a function of $\eta$ for different values of $f_{w}$. 

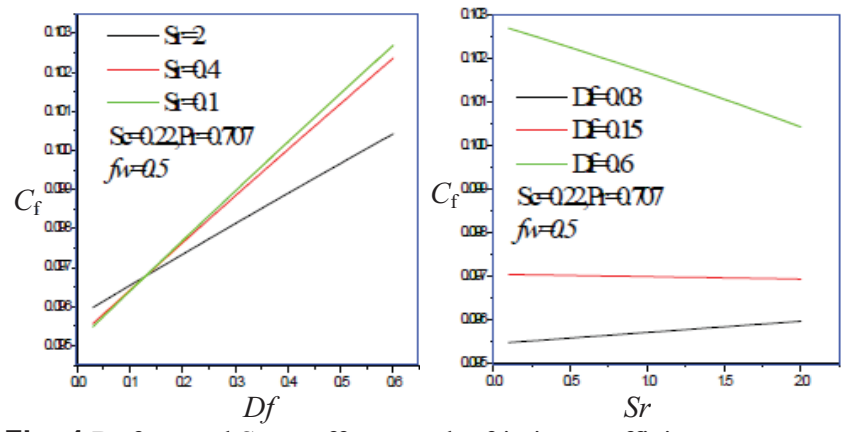

Fig. 4 Dufour and Soret effects on the friction coefficient versus: a- $D f$ for different values of $S r$, and b-. $S r$ for different values of $D f$

\subsection{Dufour and Soret effects on the Nusselt and Sherwood numbers}

Figure 5 shows the variation of Nusselt Sherwood numbers according to Dufour and Soret numbers. Fig. 5a, for $\mathrm{Sr}=2$, shows that the Nusselt number increases with increasing Dufour number, whereas for its lower values, $\mathrm{Nu}$ decreases with increasing the number of Dufour. This reveals that there, there is a value in the number of Soret, for which the Nusselt number remains invariant, regardless of Df. This corresponds to the values of Soret number of neighboring 1.48 on both sides of this issue. The Nusselt number increases almost linearly with an increase with the Soret number. This trend is more nuanced for high values of the Dufour number. For $\mathrm{Sr} \leq 1.5$ the Nusselt decreases linearly with the increase Dufour number and gives the opposite effect when $\mathrm{Sr}>1.5$. Fig. $5 \mathrm{~b}$ shows the variations of the Sherwood number for different values of Dufour and Soret numbers. This figure is the dimensionless concentration profile, represented by the Sherwood number, based on the number of Soret and Dufour numbers. Of these graphs, it appears that the influence of thermodifusion has no significant influence on mass transport, while that the Soret effect is very significant. No matter how many Soret, the Sherwood number increases with the increase Dufour number. The Sherwood number increases with the decrease in Soret. We observe the Sherwood number decreases with the increase in $\mathrm{Sr}$ whatever the values of Df.
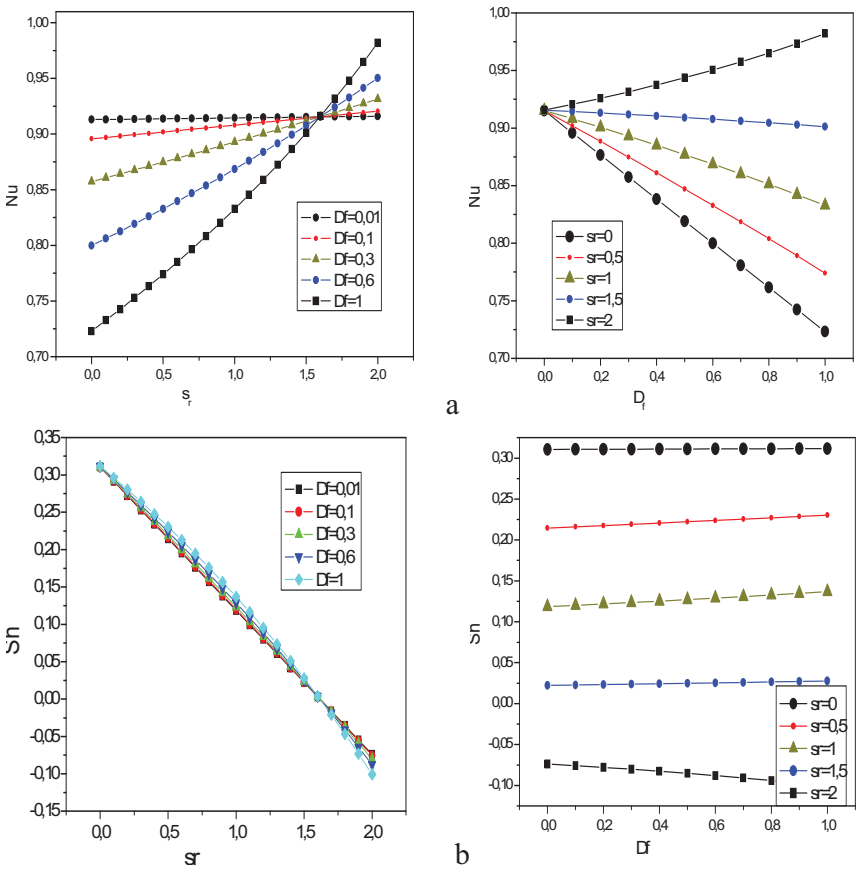

Fig. 5 Dufour and Soret effects on Nusselt and Sherwood number: a) for different values of Df, and b) for different values of $\mathrm{Sr}$

4.5 Temperature and Concentration profiles versus $\eta$ for different values of $\mathbf{G r}$ et $\mathbf{G m}$
In Fig. 6, we have plotted temperature and concentration profiles versus $\eta$ for different values of Grashoff numbers, $\mathrm{Gr}$ and $\mathrm{Gm}$. If we compare both cures, we observe that thermal and solute Grashoff effects on the dimensionless temperature are qualitatively the same. Indeed, the influence of $\mathrm{g}$ on the temperature is higher than that of $\mathrm{GM}$, for $\eta=1$. It is important to remind that in both cases, dimensionless temperature decreases with increasing both parameters Grashoff. However we are seeing the opposite phenomenon, which was preceded later: that is to say that the influence of Gm is higher than that of Gr.
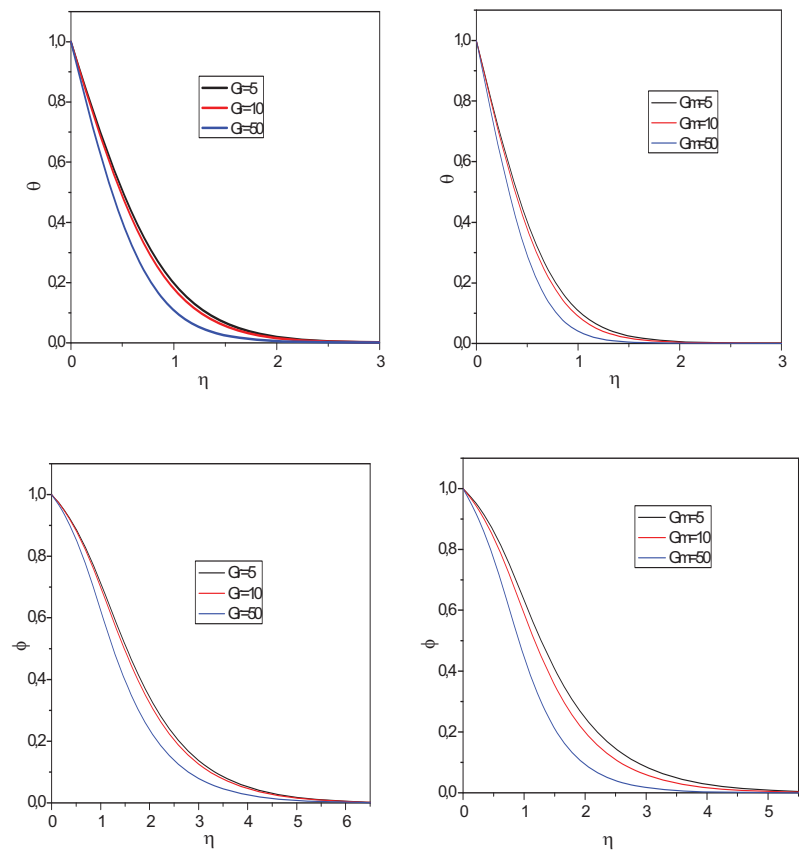

Fig. 6 Temperature and Concentration profiles versus $\eta$ for different values of $\mathrm{Gr}$ et $\mathrm{Gm}$

\subsection{Velocity, temperature and concentration profiles versus $\eta$ for different locations}

The dynamics, Thermal and mass field evolutions are represented, for different positions in the plate, in Fig. 7. We notice disturbance velocities at the lower half of the plate and begin to level off on its second half. This is the phenomenon of entrance into the plate. We note again that the velocity increases more and more when we rise on the top half of the plate. Fig. $7 \mathrm{~b}$ reveals a phenomenon of cooling of the lower part of the plate and the warming of its upper. In Fig. 7c there are fluctuations of the dimensionless mass fraction near the plate entrance. Then we joined the same remarks mentioned in paragraph 4.2.
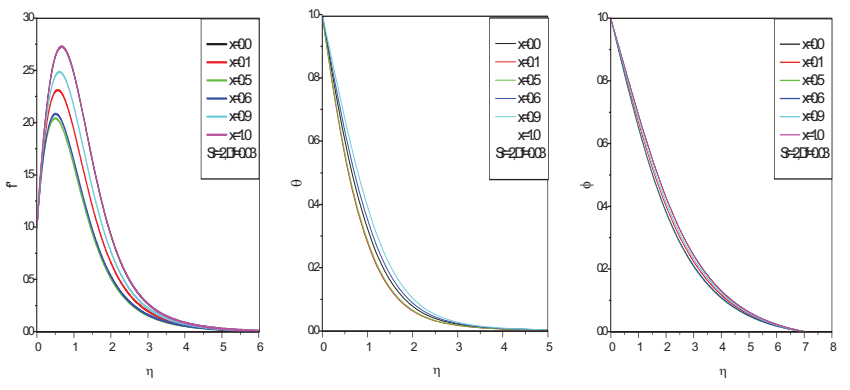

Fig. 7 Velocity, temperature and concentration profiles versus $\eta$ for different locations

4.7 Influence of inlet velocity on the profile of speed, concentration and temperature 
In Figure 8, we see that influence of the Dufour and Soret numbers on the velocity profile is insignificant for the value of the input velocity, $\mathrm{U}_{0} \geq 25 \mathrm{~m} / \mathrm{s}$, since the all curves are confused. This means that the convective transfer is much more dominant over diffusive transfer. But however, this influence affects sensibly their stability for low speeds, $\mathrm{U}_{0} \leq 0.0019 \mathrm{~m} / \mathrm{s}$. This influence is reflected in the significant instability of the velocity field for the couple $(\mathrm{Sr}, \mathrm{Df})=(0.1,0.6)$. In Fig.9 we note that the influence of the effects of Soret and Dufour on concentration and temperature profiles are almost invariant to the values of the input velocity, respectively, higher or equal critical $\mathrm{U}_{0}$. But we must also note the effects of Dufour and Soret affecting a more sensitive these profiles in the case of the minimum speed $\mathrm{U}_{0} \leq 0.0019$. This reflects that the profiles of concentration, temperature velocity are unstable in this range of speeds $\mathrm{U}_{0}$ solely for the couple $(\mathrm{Sr}=0.1, \mathrm{Df}=0.6)$. Comparing the results of the paragraph $4.3,4.4$ and 4.7 , we constant the thermo diffusion is important than the molecular diffusion.
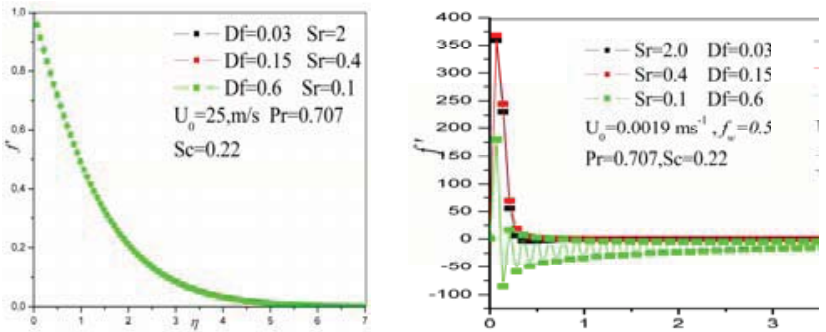

Fig. 8 variation in the speed profile based on $\mathrm{Sr}$ and $\mathrm{Df}$ for: $\mathrm{a}-\mathrm{U}_{0}=25$ $\mathrm{ms}^{-1}$ and $\mathrm{b}-\mathrm{U} 0=0.0019 \mathrm{~ms}^{-1}$
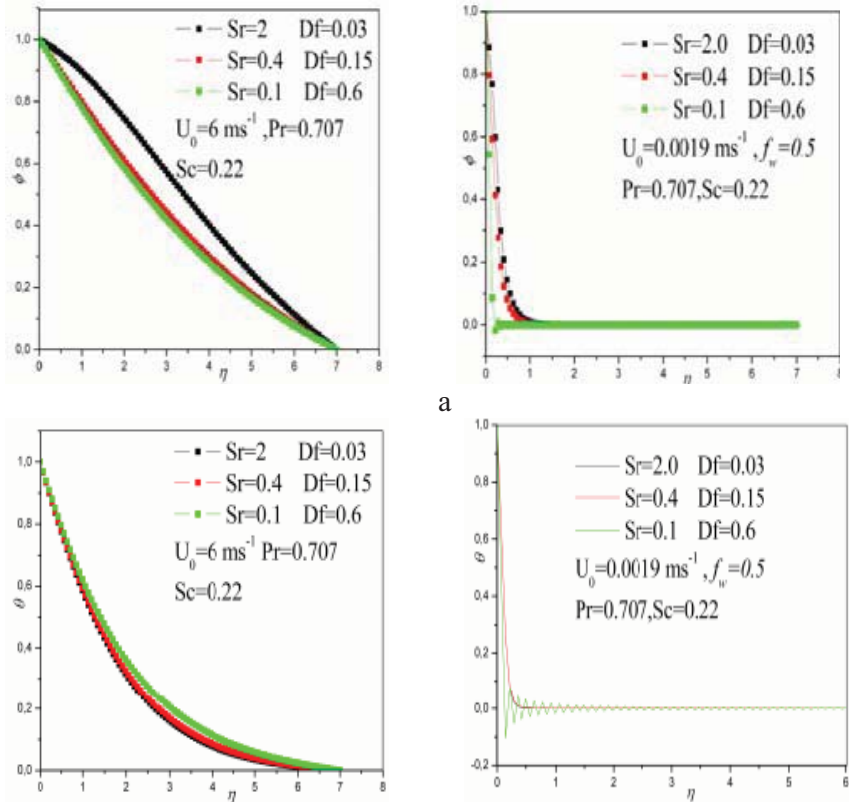

a

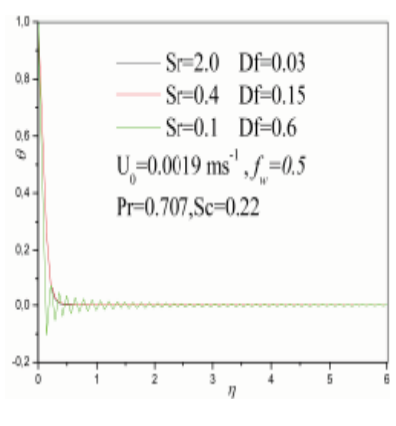

Fig. 9 variation in the concentration and temperature profiles based on ( $\mathrm{Sr}-\mathrm{Df}$ ) for: a) concentration profiles b) temperature profiles

\section{CONCLUSION}

The effect of the Soret and Dufour parameters on free convection along a vertical surface in a fluid saturated Darcy porous medium has been investigated. It was found that those two parameters do not influence the velocity, temperature and concentration fields, but rather only tend to increase the mass and energy flux due to the added contributions. The present study has shown that the suction parameter stabilizes the velocity, thermal and concentration boundary layer growth. This analyze highlight clearly the effects of the Dufour and Soret numbers on the velocity, thermal and concentration profiles and they should not be neglected.

\section{NOMENCLATURE}

$c_{p} \quad$ specific heat at constant pressure $(\mathrm{J} / \mathrm{kg} \cdot \mathrm{K})$

$h \quad$ latent heat of phase change $(\mathrm{J} / \mathrm{kg})$

$k_{\mathrm{T}} \quad$ thermal conductivity $(\mathrm{W} / \mathrm{m} \cdot \mathrm{K})$

$M \quad$ molar mass $(\mathrm{kg} / \mathrm{kmol})$

$T_{w} \quad$ Temperature at wall (K)

$T_{\infty} \quad$ Temperature at infinity (K)

$R_{g} \quad$ specific gas constant $(\mathrm{J} / \mathrm{kg} \cdot \mathrm{K})$

$T_{m} \quad$ mean temperature fluid (K)

$T \quad$ temperature (K)

$u, v \quad$ velocity components $(\mathrm{m} / \mathrm{s})$

$x, y \quad$ coordinats $(\mathrm{m})$

$C \quad$ concentration

$C_{w} \quad$ concentration at the wall

$C_{\infty} \quad$ concentration at infinity

$U_{0} \quad$ suction velocity $(\mathrm{m} / \mathrm{s})$

$\mathrm{c}_{\mathrm{s}} \quad$ concentration susceptibility

$K \quad$ permeability of porous media

Gr $\quad$ Grashoff number

Gm modified Grashoff number

$f_{w} \quad$ parameter suction

$D_{m} \quad$ mass diffusivity

$f^{\prime} \quad$ dimensionless velocity

Greek Symbols

$\alpha \quad$ thermal diffusivity $\left(\mathrm{m}^{2} / \mathrm{s}\right)$

$\theta \quad$ dimensionless temperature

Q dimensionless concentration

$\beta_{T} \quad$ coefficient of thermal expansion $\left(\mathrm{T}^{-1}\right)$

$\beta_{m} \quad$ coefficient of concentration expansion(T-1)

Superscripts

last time step

Subscripts

$0 \quad$ initial condition

w condition at the wall

$\infty \quad$ condition at the infinity

\section{REFERENCES}

Abdeldahab, E. M. and Elbarbary, E. M. E., 2001, Hall Current Effect on Magneto hydrodynamic Free Convection Flow Past a semi-infinite Vertical Plate with Mass Transfer, Int. J. Eng. Sci., 39, pp. 1641 - 1652. http://dx.doi.org/10.1111/j.1751-7176.2010.00367.x

Aouachria Z., Haddad D., Rouichi F., 2010, Soret and Dufour effects on the heat and mass transfer in porous media, 2nd International Conference on Energy Conversion and Conservation, 2nd CICME'10, April 22-25/ 2010, Hammamet, Tunisia.

Bejan A., Khair, K.R, 1985, Heat and mass transfer by natural convection in a porous medium, Int. J. Heat Mass Transfer, 28, 909918.

http://dx.doi.org/10.1016/S0735-1933(87)81002-3

Hurle D.T., Jakeman E., 1989, Soret driven thermo solutes convection Journal of fluid mechanics, 447, 667-687.

http://dx.doi.org/10.1108/09615530810879710

Jaluria, Y., 1980, Natural Convection Heat and Mass Transfer. Pergamon Press, Oxford, pp: 326.

ISBN 0080254322.

Kim, Y. J., 2004, Heat and Mass Transfer in MHD Micro polar Flow Over a Vertical Moving Porous Plate in a Porous Medium, Transport in Porous Media, 56, pp.t7-37.

http://dx.doi.org/10.1007/128_2010_10977 
Megahead, A. A., Komy, S. R, and Afify, A. A., 2003, Similarity analysis in Magneto hydrodynamics Hall Effects on Free Convection Flow and Mass Transfer Past a Semi-infinite Vertical Flat Plate, Int. Jour. Non-linear Mechanic, 38, pp.513-520.

Gr GM DJ +2 +10 2.O 0.03 7.205083 1.934014 0

Nachtsheim P.R. and Swigert P., 1969, Satisfaction of asymptotic boundary conditions in numerical solution of systems of non-linear equations of boundary layer type, NASA TN D-3004. http://dx.doi.org/10.1016/0017-9310(95)00352-5

Nasmul I. and Mahmud A., 2007, Dufour and Soret Effects on Steady Mhd Free Convection And Mass Transfer Fluid Flow Through A. A Rotating System. Journal of Naval Architecture and Marine Engineering June.

http://dx.doi.org/10.3329/jname.v4i1.915

Nield D.A. Bejan A, 2006, Convection in Porous Media, SpringerVerlag, New York, ISBN: 0387984437

Postelnicu, A., 2004, Influence of a magnetic field on heat and mass transfer by natural convection on vertical surfaces in porous media considering Soret and Dufour effects, International journal of Heat and Mass Transfer, 47, 1467-1472.

http://dx.doi.org/10.3329/jname.v6i2.3061

Rahman, M. M. and Sat Tar, M. A., 1999, MHD Free Convection and Mass Transfer Flow with Oscillatory Plate Velocity and Constant Heat Source in a Rotating Frame of Reference, Dhaka Univ. J. Sci., 9 (1), pp.63-73.

http://dx.doi.org/10.1002/ceat.200700487.1

Raptis, A. and Kafoussias, N. G., 1982, Magneto hydrodynamic Free Convection Flow and Mass Transfer Through Porous Medium bounded by an Infinite Vertical Porous Plate with Constant Heat Flux, Can. J. Phys., 60, pp. 1725-1729. http://dx.doi.org/10.1007/BF0158999

Soundalgekar, V. M., 1911, Free Convection Effects on the Stokes Problem for an Infinite Vertical Plate, ASME J. Heat Transfer, 99, pp.499- 501.

http://dx.doi.org/10.1016/0735-1933(85)90061-2

Stokes, G. G., 1856, the Effects of the Internal Friction of Fluids on the Motion of Pendulum. Trans. Comber. Phil. Soc., 9, pp. 8-106.

Weber, J. E. 1973, On thermal convection between non-uniformly heated plates. Intl J. Heat Mass Transfer.16, 961-970. http://dx.doi.org/10.1016/0022-1694(75)90039-6

Weber, J. E. 1974 Convection in a porous medium with horizontal and vertical temperature gradients. Intl J. Heat Mass Transfer17, 241-248. http://dx.doi.org/10.1016/0017-9310(74)90085-4

Weaver J. A. and Viskanta R., 1991, Natural convection due to horizontal temperature and concentration gradients two Species interdiffusion, Soret and Dufour effects, J. Hear Mass Transfer, Vol. 34, No. 12, pp. 3121-3133.

http://dx.doi.org/10.1155/2010/627475

Yih, K. A., 1999, Free Convection Effect on MHD Coupled Heat and Mass Transfer of a moving Permeable Vertical Surface, Int. Comm. Heat Mass Transfer, Vol. 26, pp.95-104.

http://dx.doi.org/10.1007/s002310100269

Yunus A. ç. Tuner R. h., 2005, Fundamentals of Thermal Fluid Sciences, p. cm.-McGraw-Hill, 2nd ed., INBS, New York http://dx.doi.org/10.1002/14356007.a17_485. 